\title{
A Bead-Based Assay for the Detection of Antibodies against Trichinella spp. Infection in Humans
}

\author{
Ruth Kahsay, ${ }^{1}$ Maria A. Gómez-Morales, ${ }^{2}$ Hilda N. Rivera, ${ }^{3}$ Isabel McAuliffe, ${ }^{3}$ Edoardo Pozio, ${ }^{2}$ and Sukwan Handali ${ }^{3 *}$ \\ ${ }^{1}$ Emory College, Emory University, Atlanta, Georgia; ${ }^{2}$ Department of Infectious Diseases, Istituto Superiore di Sanita, Rome, Italy; ${ }^{3}$ Division of \\ Parasitic Diseases and Malaria, Centers for Disease Control and Prevention, Atlanta, Georgia
}

\begin{abstract}
Human trichinellosis can be diagnosed by a combination of medical history, clinical presentation, and laboratory findings, and through detection of anti-Trichinella IgG in the patient's sera. ELISA using excretory-secretory $(\mathrm{E} / \mathrm{S})$ antigens of Trichinella spiralis larvae is currently the most used assay to detect Trichinella spp. antibodies. Beadbased assay can detect antibodies to multiple antigens concurrently; the ability to detect antibody to $T$. spiralis using a bead assay could be useful for diagnosis and surveillance. We developed and evaluated a bead assay to detect and quantify total IgG or IgG4 Trichinella spp. antibodies in human serum using $T$. spiralis E/S antigens. The sensitivity and specificity of the assay were determined using serum from 110 subjects with a confirmed diagnosis of trichinellosis, 140 subjects with confirmed infections with other tissue-dwelling parasites, 98 human serum samples from residents of the United States with no known history of parasitic infection, and nine human serum samples from residents of Egypt with negative microscopy for intestinal parasites. Sensitivity and specificity were $93.6 \%$ and $94.3 \%$ for total $\lg$ and $89.2 \%$ and $99.2 \%$ for IgG4, respectively. Twelve percent of sera from patients with confirmed schistosomiasis reacted with the IgG Trichinella bead assay, as did $11 \%$ of sera from patients with neurocysticercosis. The Trichinella spp. bead assay to detect IgG total antibody responses has a similar performance as the Trichinella E/S ELISA. The Trichinella spp. bead assay shows promise as a method to detect trichinellosis with a possibility to be used in multiplex applications.
\end{abstract}

\section{INTRODUCTION}

Human trichinellosis is a zoonotic disease caused by infective larvae of several species of the genus Trichinella. ${ }^{1,2}$ These nematode worms infect more than 100 species of mammals, birds, and reptiles worldwide. ${ }^{3,4}$ Infection of humans is acquired by ingesting raw or insufficiently cooked meat containing parasite larvae. Trichinella species are distributed globally, with human infections reported in 55 countries. ${ }^{5}$ In the United States, the median number of trichinellosis cases reported during 1997-2001 and 2008-2012 was 12 and 15 per year, respectively. ${ }^{6,7}$ Trichinellosis has traditionally been associated with the consumption of pork from domestic swine. Meat from horses and wild game (e.g., wild boar, bear, and walrus) has also been reported as infection sources. .,8-11 $^{-11}$

The clinical course of trichinellosis is highly variable, ranging from asymptomatic infection to severe and even fatal disease, depending on the number of ingested larvae and the species involved. ${ }^{12,13}$ Symptoms associated with trichinellosis include abdominal pain, diarrhea, fever, myalgia, periorbital edema, and prostration. Severe cases of trichinellosis have been associated with prolonged symptoms and cardiovascular, pulmonary, and neurological complications, which sometimes prove fatal. $^{1,13}$ Trichinellosis should be considered in patients with fever, myalgia, periorbital edema, eosinophilia, and recent consumption of poorly cooked pork or meat from horses or wild game. Diagnostic confirmation is established by positive serology with a highly specific test, seroconversion, or demonstration of larvae within the skeletal muscle. ${ }^{13}$ However, muscle biopsy tests to detect larvae have low sensitivity for light and moderate infections. ${ }^{13,14}$ Given that no pathognomonic symptoms or signs exist, suspected infections are diagnosed based on the medical history, the clinical picture, and laboratory findings such as eosinophilia and creatine phosphokinase, and through the detection of anti-Trichinella

*Address correspondence to Sukwan Handali, Division of Parasitic Diseases and Malaria, CDC, Roybal Campus, 1600 Clifton Rd., NE, Atlanta, GA 30329. E-mail: ahi0@cdc.gov
$\operatorname{lgG} .{ }^{15,16}$ Given that the excretory-secretory antigens (E/S antigens) from all currently recognized Trichinella spp. and genotypes bear a $\beta$-tyvelose epitope, which is recognized by all Trichinella-infected hosts, the antigens prepared with one species or genotype can be used to detect specific antibodies in hosts infected with other species or genotypes. ${ }^{15}$ An ELISA-based E/S antigens of Trichinella spiralis muscle larvae has a sensitivity of $99 \%$ and a specificity of $90.6 \%$ (the specificity increases to $95.6 \%$ when samples from multiparasitized persons are excluded). ${ }^{16}$ Cross-reactivity in this ELISA has been observed with sera of patients with other parasitic diseases (e.g., toxocariasis, Mansonella perstans filariasis, visceral leishmaniasis, toxoplasmosis, paragonimiasis, schistosomiasis, clonorchiasis, cysticercosis, and anisakiasis). ${ }^{16-19}$

To decrease the cross-reactivity, the use of recombinant proteins as antigens could improve the specificity of the assay. Attempts have been made to identify new Trichinella proteins as potential candidates as antigens for diagnosis. However, to our knowledge, these antigens have not been validated with large panels of serum samples from humans with Trichinella infections, and E/S antigens are still recommended in the International Commission on Trichinellosis guidelines for the diagnosis of the human trichinellosis. ${ }^{20}$ Another effort to improve specificity is to use a different conjugate recognizing other immunoglobulin classes such as $\lg$ M, IgE, or subtype of IgG, such as IgG4. ELISA and immunoblot using $\mathrm{E} / \mathrm{S}$ antigens and detecting $\lg \mathrm{M}$, IgE, and $\operatorname{lgG} 4$ in the samples have been reported to have high sensitivity and specificity. $^{21}$

We developed a bead assay using $T$. spiralis $\mathrm{E} / \mathrm{S}$ antigens to evaluate $\lg G$ and $\lg G 4$ serum antibodies of infected persons. Beyond the ability to concurrently test for reactions to multiple antigens, the bead assay platform offers a broader dynamic range than ELISA, which may translate to the assay's better sensitivity and specificity. ${ }^{22,23}$ In this study, we reported developing a bead assay using $T$. spiralis E/S antigens for the detection of total IgG or IgG4 subclass antibodies against Trichinella infection in humans. 


\section{MATERIALS AND METHODS}

Ethics statement. All samples used in this study were from clinical specimens or epidemiological studies with specific permission for future use (CDC Study Protocol Number 3580). Samples were anonymized, and the study was performed in compliance with protocols approved by the CDC's institutional review board.

Serum specimens. Serum samples $(n=110)$ from persons with a confirmed trichinellosis diagnosis based on history, clinical information, and ELISA data were tested. Within this set, 17 samples were from subjects with confirmed consumption of wild game meat and positive of larvae in muscle biopsy or PCR.

To evaluate the bead assay's specificity in detecting antiTrichinella IgG in human sera, we assembled, from our biobank, a convenience panel of serum samples obtained from patients with other parasitic infections $(n=140$, consisting of infections by Schistosoma haematobium, S. mansoni, Taenia solium, Toxocara spp., Strongyloides stercoralis, Echinococcus granulosus, Entamoeba multilocularis, Wuchereria bancrofti, Plasmodium falciparum, and Entamoeba histolytica). These parasitic infections were diagnosed by reference assays used at CDC. This panel of serum samples was combined with 98 samples obtained from healthy residents of the United States with no history of parasitic infections and nine samples acquired from residents of Egypt with negative stool microscopy for intestinal parasites.

In addition to these samples, a negative control serum pool was prepared by combining five serum samples from U.S. citizens with no international travel history. A positive control serum pool was similarly made by combining serum samples from five U.S. citizens with confirmed trichinellosis. These two controls were included in each plate for acceptance or rejection of the assay results. These controls were also used for determining the reproducibility of the assay.

Preparation T. spiralis E/S antigens. Trichinella spiralis E/S antigens were obtained from Istituto Superiore di Sanita (Rome, Italy). Briefly, the antigens were prepared by modifying previously described methods. ${ }^{15,24-26}$ Muscle larvae were recovered from mice by $\mathrm{HCl}$-pepsin (A. Costantino \& C.S.p.A., Turin, Italy) digestion according to ISO 18743: 2015 and then were washed seven times in sterile saline. Larvae were then placed into a 250-mL flask with RPMI 1640 medium (SigmaAldrich, St. Louis, MO) supplemented with $10 \mathrm{mM} \mathrm{4-(2-}$ hydroxyethyl)-1-piperazineethanesulfonic acid (HEPES buffer), $10,000 \mathrm{IU} / \mathrm{mL}$ of penicillin, $7,500 \mathrm{IU} / \mathrm{mL}$ of streptomycin, and $2.5 \mu \mathrm{g} / \mathrm{mL}$ of amphotericin $\mathrm{B}$, and incubated for 18 hours at $37^{\circ} \mathrm{C}$ in $5 \% \mathrm{CO}_{2}$. After 18 hours, viable larvae were identified by microscopy, and then larvae were collected by sedimentation. The supernatant was concentrated $\times 100$ by ultrafiltration in an Amicon cell with a 10-kDa membrane filter for 1 hour and then stored at $-20^{\circ} \mathrm{C}$ until use.

Bead-based assay development. Protein coupling to MagPlex magnetic beads. Coupling of E/S proteins/peptides to MagPlex magnetic microspheres (Luminex, Austin, TX) was carried out using 1-ethyl-3-(3-dimethylaminopropyl) carbodiimide hydrochloride (EDC)- $N$-hydroxysulfosuccinimide (Sulfo-NHS). ${ }^{22,27,28}$ We optimized the coupling buffer and amount of antigen used. The beads were briefly washed and activated in either $50 \mathrm{mM}$ 2-( $N$-morpholino)-ethanesulfonic acid pH 5 (MES) buffer containing $0.1 \%$ Tween-20 or $50 \mathrm{mM}$ $\mathrm{pH} 8.5$ borate buffer containing $0.9 \%$ sodium chloride and
$0.1 \%$ Tween-20. After 30 minutes of activation using endover-end mixing in the dark with Sulfo-NHS and EDC, the beads were washed twice with MES or borate buffered saline. The activated beads were then transferred to a new tube and washed once more. Beads were resuspended in the MES or borate buffer without Tween-20, and different amounts of protein were added with $1.25 \times 10^{6}$ beads. The total volume of the reaction was brought to $500 \mu \mathrm{L}$ with MES or borate buffer without Tween-20. The coupling was performed for 3 hours in the dark at room temperature by end-over-end mixing. Beads were incubated with blocking buffer (buffer containing $10 \mathrm{mM} \mathrm{Na}_{2} \mathrm{HPO}_{4}$ at $\mathrm{pH} 7.2 \%$ and $0.9 \% \mathrm{NaCl}$ [PBS] $+1 \%$ bovine serum albumin $[\mathrm{BSA}]+0.1 \%$ sodium azide $\left[\mathrm{NaN}_{3}\right], \mathrm{pH}$ 7.4) for 30 minutes. The coupled beads were stored at $4^{\circ} \mathrm{C}$ in PBS $+1 \% \mathrm{BSA}+0.1 \% \mathrm{NaN}_{3}+0.1 \%$ Tween- $20+$ phenylmethylsulfonyl fluoride $(1: 500)+$ pepstatin $(1: 1,000)+$ leupeptin $(1: 1,000)$. The concentration of the beads was determined by counting them in a hemocytometer using a $\times 20$ objective.

The optimal coupling conditions were determined by comparing the signal-to-noise $(\mathrm{S} / \mathrm{N})$ ratio of the pooled positive against the negative human sera for the different protein concentration and buffer used.

Bead-based immunoassay. Fifty microliters of the working MagPlex microsphere (Luminex, Austin, TX) coupled with protein mixture (50 beads $/ \mu \mathrm{L}$ in PBS $+0.3 \%$ Tween- $20+5 \%$ instant nonfat dry milk) and $50 \mu \mathrm{L}$ of diluted sera (1:100 in PBS $+0.3 \%$ Tween-20 + 5\% instant nonfat dry milk) were added into each well of Costar 96-well black, round-bottom plates (Fisher Scientific, Cat.\# 3,792, Hannover Park, IL). After 30 minutes incubation on a Titer Plate Shaker (Lab-Line, model \#4625, Melrose Park, IL) at room temperature with shaking at $\sim 800 \mathrm{rpm}$, the beads were washed using the Biotek Magnetic Washer EL $\times 50$ (2 minutes of magnetic separation followed by two cycles of dispensing $100 \mu \mathrm{L}$ of PBS $+0.3 \%$ Tween-20 and a 40-second soak before aspiration). The complex of antibody and coupled beads was incubated with $50 \mu \mathrm{L}$ of mouse antihuman IgG (Fc)-biotin (Cat. \# 9,042-08, Southern Biotech, Birmingham, AL) diluted 1:200 in PBS + 1\% $\mathrm{BSA}+0.1 \% \mathrm{NaN}_{3}$ or mouse antihuman lgG4 (Fc)-biotin (Cat. \# A10663, Invitrogen, Carlsbad, CA). After 30 minutes of incubation, the beads were washed as earlier. Fifty $\mu \mathrm{L} /$ well of $R$ phycoerythrin-labeled streptavidin conjugate (Invitrogen, Cat. \# S866) at a 1:250 dilution in PBS + 1\% BSA + 0.1\% $\mathrm{NaN}_{3}$ was added to the wells and incubated for 30 minutes. After a final wash, the beads were resuspended in $100 \mu \mathrm{L} /$ well of PBS + $1 \% \mathrm{BSA}+0.1 \% \mathrm{NaN}_{3}$. The median fluorescence intensity minus blank (MFI) from each well was determined using the BioPlex manager software, version 6.02 (Bio-Rad, Hercules, CA) on a Luminex 100 platform.

Reproducibility of Trichinella spp.-bead assay. Inter-assay variability was determined for one medium-reactive control sample by testing the sample in triplicate per run for 27 different plates for IgG total antibody responses following EP5A2 (National Committee for Clinical Laboratory Standard) guidelines. ${ }^{29}$ The acceptable coefficient variation is less than $15 \% .{ }^{30}$ The medium reactive sample was constructed by diluting strong pooled positive sera into pooled negative human sera to get an MFI in the moderate range ( 5,000-10,000 MFI).

Data analysis. Data were tabulated and analyzed using Microsoft Excel (Microsoft, Redmond, WA). Determination of the cutoff value and assay performance was performed using $\mathrm{R}$ statistical software version 3.3.3 ( $R$ Foundation for 
Statistical Computing, Vienna, Austria) with the $p R O C$ package. Statistical analysis for two correlated ROC curves was carried out using DeLong's test. ${ }^{31}$

\section{RESULTS}

Determination of optimal antigen-coupling conditions. The different coupling condition results are presented in Table 1. Based on the S/N ratio, the T. spiralis E/S antigens' optimal coupling to the magnetic beads for the detection of total IgG was carried out with $1 \mu \mathrm{g}$ of E/S antigens per $1.25 \times$ $10^{6}$ beads in MES buffer, pH 5.0. For the IgG4 assay, the optimal S/N coupling used $12.5 \mu \mathrm{g}$ E/S protein per $1.25 \times 10^{6}$ beads in MES buffer. The couplings in $\mathrm{pH} 8.5$ borate buffer did not yield a good $\mathrm{S} / \mathrm{N}$ ratio. For the detection of the IgG4 antibody, $125 \mu \mathrm{g}$ of E/S antigen gave an optimal reproducibility.

After determining the optimal coupling conditions, the intraand inter-plate assay variations were determined (data not shown). The assay had a coefficient of variation of $11 \%$ for intraplate and $13 \%$ for inter-plate variation for detecting total IgG antibody to $T$. spiralis.

Bead assay performance. When detecting total IgG, the cutoff was $77 \mathrm{MFI}$ as determined by ROC analysis, with a sensitivity of $93.6 \%(103 / 110 ; 95 \% \mathrm{Cl}$ : $88-98)$ and a specificity of $94.3 \%(233 / 247 ; 95 \% \mathrm{Cl}$ : 96-100) (Figure 1). When detecting IgG4, the cutoff was $21.6 \mathrm{MFI}$ determined by ROC analysis, with a sensitivity of $89 \%(84 / 94 ; 95 \% \mathrm{Cl}$ : 80-94) and a specificity of $99.2 \%(129 / 130 ; 95 \% \mathrm{Cl}$ : 97-100) (Figure 1). There was no statistically significant difference $(P=0.07)$ in the performance of Trichinella spp. bead assay on the detection of total IgG or IgG4 antibody responses.

The total IgG bead assay exhibited cross-reactivity with serum from patients testing positive for antibodies to S. mansoni (4/33), T. solium (7/64), Toxocara spp. (1/11) and $P$. falciparum (1/3). The lgG4 bead assay has cross-reactivity with the only $S$. haematobium serum positive (Table 2 ).

Positive Trichinella spp. bead assay results were obtained for $82 \%$ (14/17) of sera from patients with a confirmed history of wild game meat consumption and positive by muscle biopsy or PCR. The three sera in this group that tested negative by bead assay were also negative by the reference ELISA. Two of the three negative sera came from subjects who tested positive for larvae's presence by muscle biopsy.

\section{DISCUSSION}

The novel bead assay based on $T$. spiralis E/S antigens described here performed well for differentiating persons with trichinellosis from negative subjects. Although this is a novel assay for human specimens, a $T$. spiralis E/S antigen beadbased assay has been developed and showed a high level of agreement with the $T$. spiralis E/S antigen-based ELISA in detecting antibodies against $T$. spiralis in sera of both experimentally and naturally infected swine..$^{32,33}$ The human $T$. spiralis bead assay has similar performances with that of porcine trichinellosis bead assay (87\% sensitivity and 95\% specificity).

Generally, the bead assay showed better sensitivity than many commercially available ELISAs, although head-to-head comparisons have not been made. Three commercial E/S antigen-based ELISAs used in a trichinellosis outbreak showed a sensitivity of $59-64 \% .^{34}$ By contrast, ELISAs using larval crude antigen extracts have similar results to the bead assay, ${ }^{35}$ but no specificity was reported. Although the bead assay has better sensitivity than the reported commercial ELISAs, it appears to be comparable to standard E/S antigenbased ELISA developed by Sun et al. for the examination of sera collected from trichinellosis patients, which has a reported sensitivity and specificity of $100 \%$ and $96.9 \%$, respectively. ${ }^{19}$ A similar ELISA based on E/S antigen developed to test sera of patients with confirmed trichinellosis from an outbreak in Italy has a reported sensitivity of $98.7 \%$ and a specificity of $98.4 \% .^{15}$

Of the seven serum samples from confirmed trichinellosis cases tested negative by bead assay, three negative sera came from the 17 samples with a muscle biopsy or PCR tested. These three negatives were also negative by the CDC reference assay. Two of the false negatives showed Trichinella spp. larvae on muscle biopsy. These false negatives could have resulted from serum collection in the early stage of infection. Early in infection, antibodies against E/S antigens harvested from $T$. spiralis muscle larvae are rarely detected, most likely because patients had yet to develop an immune response to the muscle larvae. Swine sera collected in earlystage infection ( $<50$ days postinfection) has significantly lower antibody levels than later days postinfection. Commercial ELISA tested against the same swine sera resulted in similarly low responses to the bead assay. ${ }^{27}$

The last false-negative sera of these 17 samples came from a human trichinellosis outbreak sera collection in which

TABLE 1

Optimal coupling conditions for Trichinella spp. E/S antigens as measured by the mean fluorescence intensity of the sample minus a background blank (median fluorescence intensity).

\begin{tabular}{|c|c|c|c|c|c|c|c|}
\hline \multirow[b]{2}{*}{ Buffer } & \multicolumn{3}{|c|}{ lgG total } & \multicolumn{4}{|c|}{$\operatorname{lgG}_{4}$} \\
\hline & Borate & & & Borate & & MES & \\
\hline$\mu \mathrm{g}$ of $\mathrm{E} / \mathrm{S}$ antigens & 12.5 & 1.0 & 12.5 & 12.5 & 1.0 & 12.5 & 125.0 \\
\hline \multicolumn{8}{|l|}{ Serum samples* } \\
\hline Trichinellosis & 7,116 & 14,842 & 29,979 & 54.7 & 1,054 & 2,290 & $1,261.7$ \\
\hline Cysticercosis & 5.3 & 4.2 & 28.3 & 2 & 27.7 & 38.7 & 22 \\
\hline Toxocariasis & 69.2 & 55.2 & 297.2 & 10 & 96.2 & 168.2 & 105.2 \\
\hline Negative control & 17.3 & 10.2 & 49.7 & 2.5 & 20 & 28.7 & 19.8 \\
\hline $\mathrm{S} / \mathrm{N}$ to cysticercosis & 1,342 & 3,534 & 1,059 & 27 & 38 & 59 & 57 \\
\hline $\mathrm{S} / \mathrm{N}$ to toxocariasis & 103 & 268 & 101 & 6 & 11 & 14 & 12 \\
\hline $\mathrm{S} / \mathrm{N}$ to negative control & 411 & 1,455 & 603 & 22 & 53 & 80 & 64 \\
\hline
\end{tabular}

excretory-secretory; $\mathrm{S} / \mathrm{N}=$ signal to noise

* Serum samples from persons with different infectious diseases or healthy persons. 


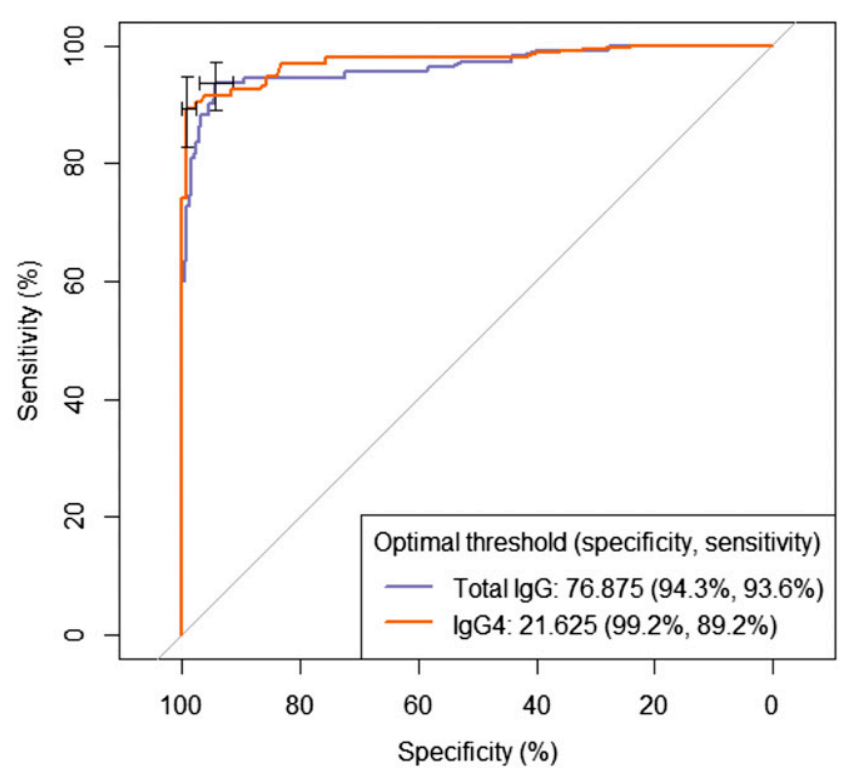

Figure 1. ROC curves of Trichinella spp. bead assay to detect $\operatorname{lgG}$ total, and $\operatorname{lgG}_{4}$ responses were constructed using $p R O C$ package of in $R$ software version 3.3.3. This figure appears in color at www.ajtmh.org.

specimen donors had documented consumption of sausage made from Trichinella positive pork and also showed symptoms and signs of trichinellosis, but no larvae were detected on muscle biopsy. This subject is the only one with negative serology, whereas the rest had positive serology. As seroconversion has been observed up to 60 days postinfection in persons with light infections, this subject, which was tested 30 days after exposure, most likely did not produce enough antibody responses to the E/S antigens for detection.

Nonspecificity in the bead assay described here was observed mostly among sera from patients with schistosomiasis, cysticercosis, and toxocariasis, as well as with one parasite negative serum sample. The use of E/S antigens in an ELISA might give rise to false-positive results because of the presence of antigenic components shared with other helminths and other organisms. ${ }^{36}$ Detection of IgG4, as opposed to total IgG, increased the specificity of the assay, with no detectable cross-reactivity against sera from individuals with other parasitic infections. However, it may not be feasible for

TABLE 2

Cross-reactivity of Trichinella spp. bead assay

\begin{tabular}{lll}
\hline & \multicolumn{2}{c}{ Cositive/number of sera (\%) } \\
\cline { 2 - 3 } \multicolumn{1}{c}{ Condition } & \multicolumn{1}{c}{ lgG total } & lgG4 \\
\hline Negative human sera (USA) & $1 / 98(1)$ & $0 / 98$ \\
Negative human sera (Egypt) & $0 / 9$ & $0 / 9$ \\
Schistosomiasis (S. haematobium) & $0 / 7$ & $1 / 1(100)$ \\
Schistosomiasis (S. mansoni) & $4 / 33(12)$ & $0 / 7$ \\
Neurocysticercosis & $7 / 64(11)$ & $0 / 5$ \\
Toxocariasis & $1 / 11(9)$ & $0 / 1$ \\
Strongyloidiasis & $0 / 4$ & $0 / 4$ \\
Echinococcosis (E. granulosus) & $0 / 9$ & $0 / 4$ \\
Echinococcosis (E. multilocularis) & $0 / 1$ & n.d. ${ }^{*}$ \\
Filariasis & $0 / 6$ & $0 / 2$ \\
Malaria (Plasmodium falciparum) & $1 / 3(33)$ & n.d. \\
Amebiasis (Entamoeba histolytica) & $0 / 2$ & n.d. \\
\hline${ }^{*}$ n.d. = not determined. & &
\end{tabular}

the IgG4-based bead assay to be used on a large scale, as it needs a larger amount of E/S antigens for coupling, thereby increasing the cost of the assay. In addition, the IgG4 bead assay has a much lower $\mathrm{S} / \mathrm{N}$ ratio than the bead assay to detect lgG total antibody.

The sera selection for this study was less than ideal as there was no matching of cases and controls sera due to using sera available in our biobank. Also, some defined trichinellosis positive sera were established using an earlier, less specific ELISA test and may not be true positives. Unfortunately, confirmation by muscle biopsy and PCR was only available for 17 of the defined positive samples.

Nonspecificity to other parasitic infections could be improved. Previous studies show that anti-Trichinella IgG and IgG4 antibodies in Western blot platform seem to be specific. Analysis of E/S antigens revealed that these antigens are a complex mixture, and some antigens may give rise to falsepositive results. Only a triple-band pattern of IgG responses (53-72 kDa) and lgG4 response directed to a 45-kDa component of the E/S antigens was Trichinella specific. ${ }^{15,36} \mathrm{De}-$ velopment of recombinant proteins of those markers of Trichinella spp. infection, in combination with a bead assay, could improve the performance of Trichinella spp. bead assay.

In conclusion, the Trichinella spp. bead assay has good performance. In a situation where multiplexing is involved, as in community surveys, the bead assay platform's advantage is obvious. Besides the possibility of testing several different antigens in a single well concurrently, the amount of antigen needed per scale of coupling is low, so it has cost-saving value. Because of the wide range of detection, it has a potential to have a better sensitivity. This sensitivity could be improved also by the multiplexing capability. The real disadvantage for working with the bead-based assay is the need for a specific equipment so that this assay is not field friendly. It is desirable to continue searching for new recombinant antigens or finding ways to improve specific lgG4 detection to improve the assay's specificity.

Received December 9, 2020. Accepted for publication February 3, 2021.

Published online March 29, 2021.

Authors' addresses: Ruth Kahsay, Emory College, Emory University, Atlanta, GA, E-mail: ruth.a.kahsay@emory.edu. Maria A. Gómez-Morales and Edoardo Pozio, Department of Infectious Diseases, Istituto Superiore di Sanita, Rome, Italy, E-mails: mariaangeles.gomezmorales@iss.it and edoardo.pozio@iss.it. Hilda B. Rivera, Isabel McAuliffe, and Sukwan Handali, Division of Parasitic Diseases and Malaria, Centers for Disease Control and Prevention, Atlanta, GA, E-mails: igi2@cdc.gov, ibm4@ cdc.gov, and ahi0@cdc.gov.

\section{REFERENCES}

1. Dupouy-Camet J, Kociecka W, Bruschi F, Bolas-Fernandez F, Pozio E, 2002. Opinion on the diagnosis and treatment of human trichinellosis. Expert Opin Pharmacother 3: 1117-1130.

2. Murrell KD, Pozio E, 2011. Worldwide occurrence and impact of human trichinellosis, 1986-2009. Emerg Infect Dis 17: 2194-2202.

3. Pozio E, 2005. The broad spectrum of Trichinella hosts: from coldto warm-blooded animals. Vet Parasitol 132: 3-11.

4. Pozio E, Darwin Murrell K, 2006. Systematics and epidemiology of trichinella. Adv Parasitol 63: 367-439.

5. Pozio E, 2007. World distribution of Trichinella spp. infections in animals and humans. Vet Parasitol 149: 3-21. 
6. Moorhead A, Grunenwald PE, Dietz VJ, Schantz PM, 1999. Trichinellosis in the United States, 1991-1996: declining but not gone. Am J Trop Med Hyg 60: 66-69.

7. Wilson NO, Hall RL, Montgomery SP, Jones JL, 2015. Trichinellosis surveillance--United States, 2008-2012. MMWR Surveill Summ 64: 1-8.

8. Larrat S, Simard M, Lair S, Belanger D, Proulx JF, 2012. From science to action and from action to science: the Nunavik Trichinellosis Prevention Program. Int J Circumpolar Health 71: 18595.

9. Pozio E, 2014. Searching for Trichinella: not all pigs are created equal. Trends Parasitol 30: 4-11.

10. Pozio E, 2015. Trichinella spp. imported with live animals and meat. Vet Parasitol 213: 46-55.

11. Rostami A, Gamble HR, Dupouy-Camet J, Khazan H, Bruschi F, 2017. Meat sources of infection for outbreaks of human trichinellosis. Food Microbiol 64: 65-71.

12. Clausen MR, Meyer CN, Krantz T, Moser C, Gomme G, Kayser L, Albrectsen J, Kapel CM, Bygbjerg IC, 1996. Trichinella infection and clinical disease. QJM 89: 631-636.

13. Dupouy-Camet J, Bruschi F, 2007. Management and diagnosis of human trichinellosis. Dupouy-Camet J, Murrell KD, eds. FAO/ WHO/OIE guidelines for the surveillance, management, Prevention and Control of Trichinellosis. Paris, France: FAO/WHO/ OIE, 37-68.

14. Moskwa B, Bien J, Cabaj W, Korinkova K, Koudela B, Stefaniak J, 2009. The comparison of different ELISA procedures in detecting anti-Trichinella IgG in human infections. Vet Parasitol 159: 312-315.

15. Gomez-Morales MA, Ludovisi A, Amati M, Blaga R, Zivojinovic M, Ribicich M, Pozio E, 2012. A distinctive Western blot pattern to recognize Trichinella infections in humans and pigs. Int $J$ Parasitol 42: 1017-1023.

16. Gomez-Morales MA, Ludovisi A, Amati M, Cherchi S, Pezzotti P, Pozio E, 2008. Validation of an enzyme-linked immunosorbent assay for diagnosis of human trichinellosis. Clin Vaccin Immunol 15: 1723-1729.

17. Chapa-Ruiz MR, Gonzalez-Pantaleon D, Morales-Galan A, Contreras-Ramos A, Salinas-Tobon MR, Martinez YZR, 2001. A follow-up study of the human class and subclass antibody response developed against the adult stage of Trichinella spiralis. Parasite 8: S163-S167.

18. Cui J, Wang L, Sun GG, Liu LN, Zhang SB, Liu RD, Zhang X, Jiang $P$, Wang ZQ, 2015. Characterization of a Trichinella spiralis $31 \mathrm{kDa}$ protein and its potential application for the serodiagnosis of trichinellosis. Acta Trop 142: 57-63.

19. Sun GG, Liu RD, Wang ZQ, Jiang P, Wang L, Liu XL, Liu CY, Zhang $X$, Cui J, 2015. New diagnostic antigens for early trichinellosis: the excretory-secretory antigens of Trichinella spiralis intestinal infective larvae. Parasitol Res 114: 4637-4644.

20. Bruschi F, Gomez-Morales MA, Hill DE, 2019. International Commission on Trichinellosis: recommendations on the use of serological tests for the detection of Trichinella infection in animals and humans. Food Waterborne Parasitol 14: e00032.

21. Calcagno MA, Forastiero MA, Saracino MP, Vila CC, Venturiello SM, 2017. Serum IgE and IgG4 against muscle larva excretory- secretory products during the early and late phases of human trichinellosis. Parasitol Res 116: 2933-2939.

22. Anderson JP et al., 2015. Development of a Luminex bead based assay for diagnosis of toxocariasis using recombinant antigens Tc-CTL-1 and Tc-TES-26. PLoS Negl Trop Dis 9: e0004168.

23. Lammie PJ, Moss DM, Brook Goodhew E, Hamlin K, Krolewiecki A, West SK, Priest JW, 2012. Development of a new platform for neglected tropical disease surveillance. Int $J$ Parasitol 42: 797-800.

24. Gamble HR, Anderson WR, Graham CE, Murrell KD, 1983. Diagnosis of swine trichinosis by enzyme-linked immunosorbent assay (ELISA) using an excretory--secretory antigen. Vet Parasitol 13: 349-361.

25. Gamble HR, Rapic D, Marinculic A, Murrell KD, 1988. Evaluation of excretory-secretory antigens for the serodiagnosis of swine trichinellosis. Vet Parasitol 30: 131-137.

26. Murrell KD, Anderson WR, Schad GA, Hanbury RD, Kazacos KR, Gamble HR, Brown J, 1986. Field evaluation of the enzymelinked immunosorbent assay for swine trichinosis: efficacy of the excretory-secretory antigen. Am J Vet Res 47: 1046-1049.

27. Hermanson GT, 2008. Bioconjugate Techniques. Burlington, MA: Academic Press.

28. Rascoe LN, Price C, Shin SH, McAuliffe I, Priest JW, Handali S, 2015. Development of Ss-NIE-1 recombinant antigen based assays for immunodiagnosis of strongyloidiasis. PLoS Negl Trop Dis 9: e0003694.

29. Tholen DW, Kallner A, Kennedy JW, Krouwer JS, Meier K, 2004. Evaluation of Precision Performance of Quantitative Measurement Methods; Approved Guidelines, 2nd edition. Wayne, PA: NCCLS25. NCCLS document EP5-A2.

30. Administration FaD, 2001. Guidance for Industry. Bioanalytical Method Validation. Center for Drug Evaluation and Research, ed. Rockville, MD: U.S. Department of Health and Human Services.

31. Robin X, Turck N, Hainard A, Tiberti N, Lisacek F, Sanchez JC, Muller M, 2011. pROC: an open-source package for R and S+ to analyze and compare ROC curves. BMC Bioinformatics 12: 77 .

32. Bokken GC, Bergwerff AA, van Knapen F, 2012. A novel beadbased assay to detect specific antibody responses against Toxoplasma gondii and Trichinella spiralis simultaneously in sera of experimentally infected swine. BMC Vet Res 8: 36

33. van der Wal FJ, Achterberg RP, Kant A, Maassen CB, 2013. A bead-based suspension array for the serological detection of Trichinella in pigs. Vet $J$ 196: 439-444.

34. Moskwa B, Bien J, Cabaj W, Korinkova K, Koudela B, KacprzakE, Stefaniak J, 2006. The estimation of different ELISA procedures for serodiagnosis of human trichinellosis. Wiad Parazytol 52: 231-238.

35. van Knapen F, Franchimont JH, Verdonk AR, Stumpf J, Undeutsch K, 1982. Detection of specific immunoglobulins ( $\operatorname{lgG}, \lg M, \lg A, \lg E$ ) and total $\lg E$ levels in human trichinosis by means of the enzyme-linked immunosorbent assay (ELISA). Am J Trop Med Hyg 31: 973-976.

36. Pinelli E, Van Der Lugt G, Homan W, Van Der Giessen J, Kortbeek LM, 2001. Antigen recognition by lgG4 antibodies in human trichinellosis. Parasite 8: S168-S171. 